CARDIOVASCULAR

CLINICAL TRIALS

IN AFRICA

\title{
Cardiovascular clinical trials in Africa: opportunities and challenges
}

\section{Marli Terblanche and Lesley Burgess}

TREAD Research/Division of Cardiology, Department of Internal Medicine, Tygerberg Hospital and University of Stellenbosch,

Parow, South Africa

\section{Address for correspondence:}

Marli Terblanche

Division of Cardiology

Tygerberg Hospital

Parow

7500

South Africa

Email:

marli@treadresearch.com

\section{INTRODUCTION}

According to Whitworth, health research is essential for the development of low- and middle-class countries ${ }^{(1)}$ and, in a developing country like South Africa, contributes to overall healthcare "by identifying the causes of problems, facilitating diagnosis, improving the efficiency and effectiveness of care, and promoting good policy-making. It also supports the training of competent health professionals of all kinds, and contributes to global knowledge about locally, as well as generally, prevalent diseases in terms of prevention and treatment".(2) Although there have been a number of initiatives designed to strengthen research capacity in Africa over the past few years, the success thereof is difficult to measure. The Thomson Reuters Global research report attempts to do so by reporting the volume of scientific publications produced by various countries over the continent. Not surprisingly, the six leading African countries by output (namely South Africa, Egypt, Nigeria, Tunisia, Algeria and Kenya) also correspond with four of the leading countries in terms of gross domestic product (GDP). However, it is clear that the continent's research output is still small and needs to be further addressed. ${ }^{(3)}$ A recent edition of the Lancet highlighted the need for South Africa to prioritise

\section{ABSTRACT}

Africa is a continent of great diversity and consists of extremely poor and remote rural regions characterised by traditional existences, as well as highly industrialised cities populated with people following an urban westernised lifestyle. This has resulted in an interesting presentation of diseases involving the cardiovascular system. On the one hand, rheumatic heart disease, cardiomyopathy and tuberculous pericarditis remain prevalent and underresearched among the black African population, while on the other hand, cardiovascular disease and its associated risk factors are starting to emerge and currently account for $10 \%$ of all deaths. Clinical trials play a crucial role in raising public awareness and promoting good policy-making; however, the continent's research output is still small. Opportunities and challenges facing African researchers need to be identified in order to develop a strategic plan for the way forward. SAHeart 201 1; 8:1 14-121

clinical research and accordingly develop a strategic plan to this effect. ${ }^{(4,5)}$ In order to be able to institute such a plan, it is essential that the various opportunities and challenges be recognised.

\section{RESEARCHAGENDA FOR CARDIOVASCULAR}

\section{DISEASE IN AFRICA}

Africa is a continent of great diversity. On the one end, it consists of extremely poor and remote rural regions with traditional existences, while on the other end, there are highly industrialised cities populated with people following an urban westernised lifestyle. As a result of this multi-ethnic population, different stages of the epidemiologic transition are evident across Africa.

According to the literature, rheumatic heart disease, cardiomyopathy and tuberculous pericarditis are the major causes for heart disease in the African population. ${ }^{(6)}$ This leads to a high mortality and morbidity in young people, crippling the economy by debilitating and killing the productive population of adults, and leaving an 
increasing number of orphans in the care of the poor and weak elderly populations. (7) A great amount of original research is needed in cardiac disorders that are either common or rare in the black African population. Implementing preventative strategies for these conditions depend on social changes, improving primary healthcare services, elimination of poverty, better housing and an enhanced knowledge of the disease process through research and development programmes that are currently constrained by staffing and budgetary restrictions. ${ }^{(6)}$

Studies in the 1970s to 1980s suggested that the prevalence of cardiovascular disease(CVD) was low in the black African population. ${ }^{(8)}$ However, CVD has since become an ever-present cause of morbidity and a leading cause of mortality in South Africa, Zimbabwe and the rest of sub-Saharan Africa, partly due to the presence of determinants and risk factors in these societies. ${ }^{(9,10)}$ In fact, CVD is the second highest cause of death in most African and low-income countries, accounting for $10 \%$ of all deaths. ${ }^{(I)}$ It is estimated that this burden will double from 1990 to 2020.(11) The effects of CVD are well documented in developed countries. However, reliable data describing the burden of this epidemic in developing countries associated with a high prevalence of malnourishment and infectious diseases is scant. Currently, developing countries contribute a greater share to the global burden of CVD than developed countries; it has been estimated that 5.3 million deaths were attributed to CVD in developed countries in 1990 compared to 8 to 9 million deaths in developing countries. ${ }^{(12)}$

In South Africa, CVD, diabetes, respiratory disease and cancer have been heralded as the "silent killers" of this socially and culturally diverse population. One in three men and $50 \%$ of all women are overweight.(13) Increasing urbanisation and resultant unhealthy lifestyles, lead to the appearance of various chronic diseases of lifestyle risk factors such as hyperlipidaemia, diabetes, atherosclerosis, obesity, hypertension and an increasing tobacco addiction. Amongst these, CVD is the most common cause of death for people age 45 and older. ${ }^{(13)}$

The public health system, already under pressure to manage the acute care of infectious diseases such as HIVIAIDS and tuberculosis, battles to deal with the effective treatment and prevention of CVD, diabetes and cancer due to limited financial resources. This, combined with limited public awareness of these conditions, results in these diseases often going unnoticed. Clinical trials play a crucial role in raising public awareness and educating the public about identifying risk factors and early signs of chronic lifestyle diseases. (13)

\section{LACK OF CAPACITY FOR HEALTH RESEARCH}

Undertaking clinical trials in Africa is far from easy. For a start, many of the African countries have an environment that is not conducive to research and "the legislative framework has not kept pace with trends in research, such as genetics research, ethical conduct of clinical trials, material exchange, and intellectual property rights".(I) Inclusion of large numbers of patients of African origin is the ideal objective. This may contribute to exploring possible genetic differences and therefore different efficacy and adverse event profiles in these patients. It has been shown in Africans from South Africa that their $L p(a)$, known to be associated with increased CVD, differs from that of other ethnic groups, including Caucasians and Africans. ${ }^{(14)}$ In addition, there are a number of other factors that need to be considered:

In most medical schools, including those in South Africa, specialist training "traditionally focuses on clinical experience and skills accrual, and lacks a research focus".(15)

Most academic tertiary units struggle to remain academically active and in some countries, such as South Africa, this has been attributed to a cumulative disinvestment in publicly funded research programmes. For instance, in South Africa, the health departments of provincial governments no longer contribute to research, the National Health Laboratory Service does not provide any discount for research tests, and the Medical Research Council, despite having a mandate for maintaining and developing the country's research capacity, is chronically underfunded. . $^{(5,16)}$

The global nature of science and lack of career paths to attract and retain good researchers has resulted in many of the top African researchers moving to countries where their efforts 
and talents are better rewarded and better appreciated. ${ }^{(1)}$

According to ASSAf, the clinical research force is ageing and has been steadily declining in numbers since the early 1990s. ${ }^{(2)}$

In this age of evidence-based medicine, clinical guidelines and health policies should ideally be based on well-run, local, randomised controlled trials.

African governments need to provide the basic infrastructure for health research. ${ }^{(1)}$ There is increasing interest in and recognition of the crucial importance of strengthening the research capacity for health in Africa and to date there are several initiatives and schemes that have recently or are currently being developed. ${ }^{(1,17)}$ These include:

The African Health Research forum and University Science, Humanities and Engineering Partnerships in Africa (USHEPiA see http://www.ushepia.uct.ac.za/bg.htm).

The European and Developing Countries Clinical Trials Partnerships (EDCTP) Networks of Excellence (see http:// www.edctp.org).

The European-Union-funded Network for the Coordination and Advancement of sub-Saharan Africa-EU Science and Technology Cooperation (CAAST-Net - see http://www. caast-net.org).

The Health Research Capacity Strengthening (HRCS) initiative partnership between DIFD, IDRC, and the Wellcome Trust (see http://www.idrc.ca/cp/ev-1067| 3-20I-I-DO_TOPIC.html.

The Neglected Tropical Diseases (NTD) fellowship scheme, supported by a consortium of European Foundations (see http://www.ntd-africa.net).

The Netherlands-African partnership for capacity development and clinical interventions against poverty-related disease, the Netherlands Organisation for Scientific Research (see http:// www.nwo.nl/naccap).

The Royal Society UK science networking scheme with Ghana and Tanzania (see-http://www.royalsoc.ac.uk/funding. asp?id=68 | 6).
The Wellcome Trust African Institutions Initiative (see http:// www.wellcome.ac.uk/News/Media-office/Press-releases/2009/ WTX053482.htm).

While these various initiatives and schemes are headed in the right direction, they may fail to achieve their aims because of no coordination and duplication of effort. (I) The EDCTP, for instance, certainly did not get off to an auspicious start with the ousting of its executive director at the first annual forum and rumblings of discontent among grant applicants with regards to poor administration. ${ }^{(18)}$ Lessons from other sectors such as agriculture and education also need to be sought and discussed.

\section{LACK OF FUNDING FOR CLINICAL RESEARCH}

In 2007, the African Union members pledged to spend $1 \%$ of their GDP on research and development. ${ }^{(19)}$ According to ASSAf, 2\% of GDP is the necessary minimum investment in indigenous science and technology development, with health research receiving at least $20 \%$ of this amount. (2) Currently in South Africa, $10.8 \%$ of all government expenditure is on health and, according to the National Health Expenditure Review in 1991/1992, it was estimated that about $1.1 \%$ of this was spent on research. South Africa is spending more on research and development than before, but it is still considerable less than $1 \%$ of GDP.

Clinical researchers are thus being left with no alternative but to rely on funding from the pharmaceutical industry (whose predominant interest is aimed at profitable areas such as chronic diseases of lifestyle, mental illness and allergies) and international donors (whose predominant interest is HIV and tuberculosis). ${ }^{(16)}$ The overall effect of this is that the local needs of the countries are being bypassed. Governments from these nations accordingly need to start recognising that research funds are a "good investment". (1)

\section{PROTECTION OF PARTICIPANTS}

According to section 12(2)(c) of the constitution of South Africa Act, No 108 of 1996: "Everyone has the right to bodily and 
psychological integrity, which includes the right...not to be subjected to medical or scientific experiments without their informed consent."(20) In Africa, where cultural, political and racial factors give rise to third world conditions, special attention should still be given to obtain informed consent from rural or vulnerable communities. In South Africa, for instance, a local version of Good Clinical Practice, namely South African Good Clinical Practice guidelines (SAGCP), is used in conjunction with the International Conference of Harmonisation $(\mathrm{ICH})$ version. Much of the content of SAGCP is aimed at promoting safe research among vulnerable populations and ensuring that researchers are held accountable. This is achieved by:

Incorporating a separate section dedicated to "Research requiring additional attention" (Section 2.3) including research involving, inter alia, collectivities, indigenous medical systems, vulnerable communities, minors, mentally disabled people, HIV/ AIDS, and women and foetuses, and

Ensuring that principal investigators are South African based scientists (Section 3.1). ${ }^{(21)}$

It is also essential that all clinical research involving human participants is approved by an independent research ethics committee. Ethical and regulatory issues, such as possible inadequacy with regards to regulatory and ethical control and overview, and varying competency of local ethics committees are frequently cited as problems characterising these bodies in developing countries. ${ }^{(22-24)}$ Such concerns were highlighted recently with regards to criticism of a clinical trial in South Africa that was published in the February 2010 edition of the New England Journal of Medicine, namely the SAPIT study. ${ }^{(25)}$ The ensuing blog on the website of the Bioethics Forum of the Hastings Centre stated that this trial had violated both ethical study design and the Declaration of Helsinki, and insinuated that research ethics review and training in South Africa was substandard. ${ }^{(26)}$ These findings were upheld by Boulle. (27) In defence, Cleaton-Jones argued that "at a structural level research ethics review in South Africa is in many cases equivalent to the US institutional review board (IRB) and Office for Human Research Protections (OHRP) oversight system, is wider reaching, and has no exclusions". (28) Nonetheless, training for research ethics committee members is essential and remains a global issue - especially in the context of third world countries.

\section{LANGUAGE BARRIER}

Africa, with its rich cultural heritage, diverse population and wealth of traditional knowledge has a lot to offer modern science, but much is lost due to language constraints. Conversely, this also poses a problem when science cannot be translated into local languages. In South Africa, for instance, there are II official languages and a population consisting of black Africans $79 \%$, white $9.6 \%$, coloured 8.9\% and Asian 2.5\%. ${ }^{(13)}$ Translations are usually required for the informed consent forms and patient information brochures, however English is acceptable for the clinical protocols, CRFs and Investigators Brochures. ${ }^{(13)}$

The translation of African languages and practices results in a great deal of local knowledge being lost, and leads to farmers and rural communities being excluded from the modernisation of science and technology, as there are no local terminology or expressions to explain or translate the two concepts. ${ }^{(19)}$ Zimbabwean scientists are currently compiling a Shona dictionary, a language currently being spoken by 9 million Zimbabweans. ${ }^{(19)}$ One of the problems with this dictionary is the inability of literal translations to adequately explain an entire concept. Furthermore, translations can only succeed when sufficient funding is available.

\section{INFORMED CONSENT}

In African communities, important concepts of informed consent such as risk-benefit ratio, fair treatment of participants and voluntary participation, are interpreted in a different way. ${ }^{(29)}$ However, the recruitment of participants in clinical trials remains essential to the success of the trial. In third world countries, participant recruitment is complicated by political, ethical, social and scientific concerns, especially when research is funded by affluent countries. ${ }^{(29)}$ According to the Declaration of Helsinki, when undertaking "medical research on human subjects, considerations related to the wellbeing of the human subject should take precedence over the interests of science and society". (30) The process of individual informed consent of subjects is, accordingly, crucial. 
Subjects from developing countries are regarded as excellent candidates for clinical trials as people in these communities tend to be poor, malnourished, illiterate and sometimes desperate. The Nuremberg Code states that "voluntary consent of the human subject is absolutely essential. This means that the person involved should have legal capacity to give consent, should be so situated as to be able to exercise free power of choice, without the intervention of any element of force, fraud, deceit, duress, overreaching, or other ulterior form of constraint or coercion".(31) However, in many African countries, personal choice is particularly limited. In particular, in African cultures the concept of personal choice or free power of choice (collectively named personhood) is vastly different from western civilisation. A person's tribe, village or social group defines ones personhood. In other words, persons exist only in relation to other persons. ${ }^{(29)}$ This complicates the process of obtaining individual informed consent, as each individual member of the community sees the community as themselves. Investigators should thus shift the consent process from individual consent to the family or community. In some cases, investigators would need to approach the community's elders for their consent before seeking consent from an individual.

According to African tradition, obtaining individual consent requires that a member of the family also gives consent to participate in medical research. This might impose a new challenge with the introduction of a waiting period. This waiting period can often be problematic as a result of the need for the individual to return to the region, with added transport costs and a loss of time. ${ }^{(29)}$ Furthermore, the possibility of misinterpretation of the consent form poses a real threat, and it may result in no permission being granted to enter the study.

Informed consent forms (ICF) often present highly complex information that needs to be understood by patients. It is important that subjects truly understand specific elements in the ICF, including the purpose of the study, the randomisation process, and risks and benefits in participating, and that participation is voluntary. Complexity of the consent form has been shown to be a major barrier to comprehension for many patients. Examples of these barriers include excessive length of the form, inadequate time to read the consent, the reading level, and the format and layout of the form. ${ }^{(32)}$ These obstacles are especially problematic for subjects with low literacy skills. According to a recent study conducted in South Africa, the readability levels of 84 ICFs used over a period of ten years were examined. The mean \pm standard deviation (SD) Flesch-Kincaid Reading Ease score for the 84 ICFs was $46.60 \pm 5.62$ (range 33.2-65.6). The mean \pm SD grade level was $12.13 \pm 1.8$ (range 8.3-14.9) using the Flesch-Kincaid formula and I $3.96 \pm 1.22$ (range 10.3-16.6) using the Gunning-Fog index. The recommended grade 8 readability level was only found in $1.2 \%$ of all the ICFs assessed. ${ }^{(32)}$

It is the investigator's responsibility to ensure that a potential trial subject is competent to fully understand the informed consent. A study conducted in a cardiology research unit in Cape Town, South Africa demonstrated that, despite adequate time and explanations being provided by researchers, participant understanding of certain research terminologies, such as randomisation and placebo, were poor. ${ }^{(33)}$ Thus more time and effort are needed for participants with lower levels of education and/or little experience of research or medical contexts. A person is deemed competent if "able to understand a therapy or research procedure, to deliberate major risks and benefits, and to make a decision in light of this deliberation". (29)

Due to malnourishment and poverty, people in developing countries do not have equal opportunities for education and are commonly considered illiterate or uneducated. These people are classified as vulnerable research subjects because they often lack the capacity or competency to give informed consent. People in vulnerable communities often do not understand the complicated protocol requirements or procedures, which results in them being unable to decide whether or not to participate in a trial.

\section{OPPORTUNITIES AND CONCERNS REGARDING} GLOBALISATION OF CLINICAL RESEARCH

In recent years, there has been a significant geographical shift in the conduct of clinical research from first world countries, such as 
the United States and Western Europe, to developing countries, including those in Africa. ${ }^{(22)}$ Tracking research in Africa remains difficult as there are few published studies reporting this. ASSAf reports that only $1 \%$ of the total spent on clinical trials worldwide is spent in Africa, with $80 \%$ of that being spent in South Africa. ${ }^{(2)}$

South Africa has seen a consistent growth in clinical research emanating from contract research organisations and pharmaceutical companies. In 200I, an estimated 400 clinical trials were being conducted in South Africa with expenditure in excess of USD I50 million. ${ }^{(13)}$ In 2008, it was estimated that this figure has increased to approximately R2.2 billion. ${ }^{(34)}$ In fact, a total of I 098 trials are currently registered in the South African National Clinical Trials Register (SANCTR), with cardiovascular diseases and diabetes mellitus accounting for just over 10\%. ${ }^{(35)}$ Key factors for the growth in clinical trials in South Africa include a history of high quality medical research, a high burden of disease and relatively drug-naïve populations. ${ }^{(36)}$

There are a number of concerns associated with this policy of globalisation, including:

Lengthy regulatory approval times.

Concern over the standard of data.

Lack of confidentiality.

Dodgy recruitment methods.

Inexperienced and inadequate ethics committees. ${ }^{(37)}$

There have also been numerous instances where pharmaceutical companies have attempted to pursue clinical trials in developing countries which would not be considered ethical or practical in a developing country. ${ }^{(38)}$ A good example of this is the Trovan meningitis study in Nigeria during 1996. ${ }^{(23)}$ Emanuel has identified three controversial issues with regards to the conduct of clinical trials in developing countries that need to be addressed, namely the standard of care to which investigational treatments should be compared, the "reasonable availability" of proven interventions demonstrated to be beneficial during the study, and the "quality of the informed consent" process. ${ }^{(39)}$ However, there have been numerous other concerns that have been raised and include:

Personnel and site issues, such as shortage of experienced, trained site staff. Very few research centres are adequately equipped to perform clinical trials, while those few who are, remain to be oversubscribed. This necessitates the need for developing an infrastructure to conduct research in Africa. Partnerships, such as the EDCTP, have been accordingly instituted to fund research in developing countries, particularly in Africa, that contribute to the development of affordable prophylactics and drugs for HIVIAIDS, tuberculosis and malaria.(18) The EDCTP believes that the best approach in training the new generation African scientists is training on the job, involving them fully in the planning and execution of the trial. In addition, challenges, such as unreliable electricity supplies and inaccessible roads, are a reality in rural and remote sites. ${ }^{(38)}$ These can impact heavily on the conduct of the trial and hence the integrity of the data. In some cases, safety of site personnel can also be problematic.

Ethical and regulatory concerns, as discussed above.

Population diversity: The increasing environmental, cultural and ethnic diversity of the research participants may pose problems in reaching conclusions - for example, angioneurotic oedema secondary to ACE-inhibitors is more common in African Americans than in white patients. ${ }^{(22,38)}$ In addition, it is important that sponsors obtain the commitment, support and understanding of local leaders, health workers and their customs. ${ }^{(38)}$ Care must be taken to ensure that the project is not perceived as "exploitative". ${ }^{(38,39)}$

Suitability of clinical research to the population: There are ethical concerns about researching drugs in a community in which the sponsor has no intention of marketing the drug. Medication that is affordable in first world countries is often too expensive for the majority of patients in developing countries; relevant strategies need to be researched for these countries. ${ }^{(38,40)}$ In addition, historically, uneducated, socioeconomically-deprived populations were "targeted for high-risk 
research, whereas promising research was offered in more privileged individuals. ${ }^{(39)}$

The potential vulnerability of the population group must be recognised and not exploited. In some instances, remuneration for study participation may be excessive. ${ }^{(40,41)}$ In addition, trial participation may be the only way in which patients can gain access to medical services and medication to which they would otherwise be denied. This finding was confirmed in a South African study where more than $90 \%$ of respondents listed this as a reason for volunteering for a clinical trial. ${ }^{(42)}$

Other local issues, such as the use of over-the-counter concomitant medications, particularly local herbal remedies, which can cause unexpected drug interactions and adverse effects. ${ }^{(38)}$

Other issues raised by Glickman include: "transparency of clinical trial results" and subsequent "protection of publication rights and access to trial data", and "genomic information in drug development". ${ }^{40)}$ Despite these challenges, there are a number of signs that developing countries are meeting the desired quality of performance. Yusuf has analysed the performance of developing countries in a number of international randomised control levels and demonstrated that the quality of data, patient attrition and overall study performance was on par with first world countries. ${ }^{(38)}$ In South Africa, for instance, a total of 37 sites were audited by the Food and Drug Administration (FDA) of America from 1994 to 2009.(43) The most common deficiencies were "failure to follow the investigational plan" (15 sites) and "inadequate and inaccurate records" (8 sites). There was a single inspection in 1997 that resulted in an "Official Action Indicated" report being generated, with the deficiency being cited as "inadequate and inaccurate records". These findings are shown in Table I, and are better than many FDA inspection findings from developed countries.

TABLE I: Classification of FDA inspection findings at South African trial sites

\begin{tabular}{|c|c|c|c|c|}
\hline & $\begin{array}{l}\text { No Action } \\
\text { Indicated }\end{array}$ & $\begin{array}{c}\text { Voluntary } \\
\text { Action } \\
\text { Indicated }\end{array}$ & $\begin{array}{c}\text { Official } \\
\text { Action } \\
\text { Indicated }\end{array}$ & Tota \\
\hline $\begin{array}{l}\text { South African } \\
\text { sites }\end{array}$ & $16(43 \%)$ & $20(54 \%)$ & I (3\%) & 37 \\
\hline
\end{tabular}

\section{CONCLUSIONS}

Clinical research in Africa is in a state of crisis and a strategic plan needs to be developed and implemented. In many ways, South Africa appears to be leading the way with regards to research output on this continent but even in South Africa there has been almost 20\% decrease in publications between 1990 to 1994 and 1996 to $2000 .^{(5)}$ The long-term future and sustainability of clinical research in Africa depends on many factors, and are adequately summarised in the brief of the ASSAf council;(2) namely

The creation of a national culture conducive to clinical research with the acceptance and promotion of clinical trials as the basis for evidence-based medicine,

Equipping and encouraging medical students to realise the importance of clinical research and evidence-based medicine in delivering effective healthcare,

Improving the accessibility and provision of funding for investigator-driven clinical research,

Ensuring the practice of ethically acceptable clinical research with adequate protection of the rights and safety of research participants, and

Promoting the interaction and collaboration of government, parastatal institutions, academia and industry with regards to research efforts and capacity-building

In addition, initiatives aimed at improving the education of the whole population regarding Western medicine must be stepped up in order to allay fears among some ethnic groups that Western medicine may be harmful and to provide reassurance that the aim of these trials is to promote the health of everyone, including the people of Africa. 
I. Whitworth JAG, Kokwaro G, Kinyanjui S, et al. Strengthening capacity for health research in Africa. Lancet. 2008;372:1590-1593.

2. ASSAf. Consensus report on Revitalising Clinical Research in South Africa; A study on clinical research and related training in South Africa. November 2009. Available from www.assaf.org.za (Accessed: 20 February 201 I).

3. Editorial. Research out of Africa. Lancet. 2010;375:1496.

4. Lawn JE, Kinney MV. Health in South Africa: Executive summary of the series. Lancet. 2009;347:2-6.

5. Gevers W. Clinical research in South Africa: a core asset under pressure. Lancet. 2009;347:760-762.

6. Commerford P, Mayosi B. An appropriate research agenda for heart disease in Africa. Lancet. 2006;367:1884-1886.

7. Kitua AY, Corrah T, Herbst T, et al. Strengthening capacity, collaboration and quality of clinical research in Africa: EDCTP Networks of Excellence. Tanzania Journal of Health Research. 2009; I I ( I):5 I-54.

8. Steyn K, Hawken S, Commerford P, et al. Risk factors associated with myocardial infarction in Africa: the INTERHEART Africa Study. Circulation. 2005; | | 2:3554-356|

9. Mbewu A. The burden of cardiovascular disease in sub-Saharan Africa. SA Heart Summer 2009; Vol 6:4-10.

10. Hakim JG, Odwee MG, Siziya S, et al. Acute myocardial infarction in Zimbabwe: the changing scene of coronary artery disease. Cent Afr J Med. 1995 Oct;4 I ( I0): 303-308.

II. Global burden of disease update 2004. WHO 2008. Geneva, Switzerland.

12. Reddy KS, Yusuf S. Emerging of cardiovascular disease in developing countries. Circulation. 1998;97;596-601.

13. Van Wyk F, Hunt P. Spirit of Africa. EPC. 54-56 Available from: http://www. pharm-olam.com/pdf/South-Africa-EPC.pdf (Accessed 25 January 201 I).

14. Oelofse A, Jooste PL, Steyn K, et al. The lipid and lipoprotein profile of the urban black South African population of the Cape Peninsula - The Brisk Study. South African Medical Journal , 1996(86): 162-166.

15. Siegfried N, Volmink J, Dhansay A. Does South Africa need a national clinical trials support unit? SAMJ. 2010;100(8):521-524.

16. Benatar SR, Vaughan CJ. Global and legal dorces shaping the research agenda and governance of research ethics. S Afr J Sci 2008; 104:439-442.

17. Sawyer A. African universities and the challenges of research capacity development. Available from http://www.codesria.org/IMG/pdf/8-SAWYERR.pdf (Accessed 20 February 2011).

18. The PLoS Medicine Editors. A new vision for clinical trials in Africa. PLoS Med 2004; I (3):e7l. Available from: http://www.plosmedicine.org/article/info: doi//0.137//journal.pmed.001007| (Accessed I8 January 201 I).

19. Dhewa C. Africa: Science must tackle local language barriers. University World News Africa Edition. Available from: http://www.universityworldnews.com/article. php?story=20 I 00409204449682 (Accessed 26 January 20 I I).

20. Statutes of the Republic of South Africa - Constitutional Law. Constitution of the Republic of South Africa Act, No 108 of 1996.

21. South African Department of Health. Guidelines for good clinical practice in the conduct of clinical trials with human participants in South Africa. 2nd ed. Pretoria: Department of Health, 2006.

22. Thiers FA, Sinskey AJ, Berndt ER. Trends in the globalisation of clinical trials. Nature Reviews Drug Discovery 2008;7(1):13-14.

23. Annas GJ. Globalised clinical trials and informed consent. The New England Journal of Medicine 2009;360(20):2050-2053.
24. Hyder AA, Watt SA, Khan AN, et al. Ethical review of health research; a perspective from developing country researchers. Journal of Medical Ethics, 2004:30:68-72.

25. Abdool Karim SS, Naidoo K, Grobler A, et al. Starting antiretroviral therapy at three points in tuberculosis (SAPIT). The New England Journal of Medicine 2010;362:697-706.

26. Philpott S, Schüklenk U. A study that should not have been done. Available from http://www.hastingscentre.org/Bioethicsforum/Post.aspx?id=4626\&blogid= $140 \# i$ xzzOnEEmxIUB (Accessed 03 March 201 I).

27. Boulle A, Clayden P, Cohen K, et al. Prolonged deferral of antiretroviral therapy in the SAPiT trial: Did we need a clinical trial to tell us that this would increase mortality? South African Medical Journal 20 10; 100:566-57I.

28. Cleaton-Jones P, Wassenaar D. Issues in Research: Protection of human participants in health research - a comparison of some US federal regulations and South African research ethics guidelines. South African Medical Journal 2010;100(11):710-716.

29. Moodley K. HIV vaccine trial participation in South Africa - An ethical assessment. Journal of Medicine and Philosophy. 2002; Vol.27,No 2,197-215.

30. World Medical Association. Declaration of Helsinki - Ethical principles for medical research involving human subjects. Available from http://www.wma.net/ en/30publications/ I policies/b3/index.html (Accessed 26 January 20 I I).

31. The Nuremberg Code (1947) In: Mitscherlich A, Mielke F. Doctors of infamy: the story of the Nazi medical crimes. New York: Schuman, 1949: xxiii-xxv.

32. Terblanche $M$, Burgess $L$ J. Examining the readability of patient-informed consent forms. Open access journal of clinical trials. 2010:2157-162.

33. Gerber B, Delport R, Burgess $L$, et al. Current understanding of informed consent forms. Presentation at the 2nd Annual Clinical Trial Conference, SACRA, September 2008.

34. Richardson M-A. Focus on Africa. African Clinical Research Organisation (ACRO) Online. November 2009. Available from www.acro.co.za. (Accessed 17 December 2009).

35. Department of Health, South Africa. South African Clinical Trial Information. Available from www.sanctr.gov.za. (Accessed 3 March 2011).

36. Kahn M, Gastrow M. Pharmacologically active clinical trials and the pharmaceutical industry. S Afr Med J 2008;98(2): | |4-1 |6.

37. Jayaraman K. Outsourcing clinical trials to India rash and risky, critics warn. Nature Medicine 2004; 10 (5):440.

38. Yusuf S. Clinical research and trials in developing countries. Statistics in Medicine, 2002;21:2859-2867.

39. Emanuel E, Wendler D, Killen J, et al. What makes clinical research in developing counties ethical? The benchmarks of ethical research. The Journal of Infectious Diseases, 0 I March 2004; 189:930-937.

40. Glickman SW, McHutchison JG, et al. Ethical and scientific implications of the globalisation of clinical research. The New England Journal of Medicine, 19 February 2009;360(8):816-823.

41. Burgess LJ, Sulzer NU, Hoosain F, et al. Patient's motivations for participating in cardiovascular clinical trials: a local perspective. Cardiovascular Journal of Africa, July/August 2004;20(4):220-223.

42. Burgess LJ, Sulzer NU, Emmanuel S. Clinical trial remuneration: The patient's perspective. South African Medical Journal , February 2008;98(2):95-97.

43. US FDA/Centre for Drug evaluation and research. Clinical investigator inspection list: for investigational new drug studies. Available www.accessdata.fda.gov/ scripts/cder/cliil/index.cfm (Accessed 03 March 20II). 